Research Paper

\title{
A nomogram to predict long-time survival for patients with Ml diseases of esophageal cancer
}

\author{
Mina Liu, Changlu Wang, Lanting Gao, Changxing Lv, Xuwei Cai ${ }^{\bowtie}$ \\ Department of Radiation Oncology, Shanghai Chest Hospital, Shanghai Jiao Tong University. 241 West Huaihai Road, Shanghai, 200030, China. \\ $\triangle$ Corresponding author: Department of Radiation Oncology, Shanghai Chest Hospital, Shanghai Jiao Tong University. 241 West Huaihai Road, Shanghai, \\ 200030, China. E-mail: birdhome2000@163.com; Telephone: 86 22200000-3603 \\ (C) Ivyspring International Publisher. This is an open access article distributed under the terms of the Creative Commons Attribution (CC BY-NC) license \\ (https://creativecommons.org/licenses/by-nc/4.0/). See http://ivyspring.com/terms for full terms and conditions.
}

Received: 2018.05.31; Accepted: 2018.08.19; Published: 2018.10.11

\begin{abstract}
Objective: To evaluate the clinicopathologic characteristics of the long-time survivals and construct a clinical nomogram using the Surveilance, Epidemiology, and End Results (SEER) database.

Materials and Methods: Information of patients diagnosed with M1 stage esophageal cancer from 2010-2014 was retrieved from SEER database. Patients with unknown information of AJCC TNM stage or metastatic sites or marital status or surgery or survival were excluded. Demographic and clinicopathologic characteristics were compared between LTS (long time survivals: patients who have survived for no less than 2 years) and STS (shorter time survivals: patients who have survived for less than 2 years). Cox regression analysis was performed to evaluate prognostic factors. A nomogram comprising demographic and clinicopathologic factors was established to predict 1-year survival and 2-year survival for patients with $\mathrm{Ml}$ diseases.

Results: A total of 2981 patients from the SEER database were included for analysis. Compared with the STS, married people and patients with well differentiated tumors or oligometastatic site were more likely to be LTS. Also, LTS were associated with significantly less bone metastasis and more surgery. The OS nomogram, which had a c-index of 0.633 , was based on the eleven variables: gender, age, marital status, $\mathrm{T}$ stage, $\mathrm{N}$ stage, histology, grade, number of important metastatic organs and primary surgery.

Conclusions: Married patients, patients with well differentiated tumors, patients with oligometastatic site, patients without bone metastasis or liver metastasis and those who underwent surgery are associated with long time survivals. We developed a nomogram predicting 1- and 2-year OS and CSS for MI stage esophageal cancer. The prognostic model may improve clinicians' abilities to predict individualized survival and to make treatment recommendations.
\end{abstract}

Key words: esophageal cancer; nomogram; long time survivals

\section{Introduction}

Esophageal cancer is the sixth most common cause of cancer deaths globally, and causes the deaths of over 400,000 people worldwide annually [1]. For the patients with M1 diseases, the population has been growing over the past 20 years [2]. Chemotherapy is the standard treatment for these patients; however, the survival is not satisfactory, with 5-year relative survival less than 5\% [3]. Very limited patients were able to survive more than two years. Our study aimed to evaluate the clinicopathologic characteristics of the long-time survivals and construct a clinical nomogram using the Surveillance, Epidemiology, and End Results (SEER) database.

\section{Materials and Methods}

This retrospective study was based on publicly available SEER database. Data were retrieved through online access using the SEER*Stat software Version 
8.2.1. The Institutional Review Board of Shanghai Chest Hospital approved this study.

\section{Data collection}

Information of patients diagnosed with M1 stage esophageal cancer from 2010-2014 was retrieved from the recent SEER-18 database. We used site codes C15.0-C15.5, C15.8 and C15.9 to identify primary esophageal cancer. The histology was determined by the International Classification of Diseases Codes (Squamous carcinoma: 8050, 8070-8074,8083; Adenocarcinoma: 8012, 8323, 8140, 8144, 8145, 8210, 8255, 8260, 8263, 8323, 8480, 8481, 8490, 8574; Others: 8000, $8010,8013,8020,8033,8041,8042,8051,8046,8200$, $8244,8246,8247,8560,8936)$. We limited this study to patients diagnosed between 2010 and 2014 as detailed information about site-specific metastasis was not recorded before 2010. Patients with unknown information of AJCC TNM stage or metastatic sites or marital status or surgery or survival were excluded. We also excluded patients whose information was collected from autopsy and death certificates. Individual data retrieved for each case included age at diagnosis, gender, race, year of diagnosis, tumor histology, grade, TNM stage, marital status, site-specific metastasis, surgery, cause-specific death classification, vital status and survival months. Patients were divided into two groups, the long-time survivals (LTS) group and the shorter time survivals (STS). We defined patients who survived no less than 2 years as the LTS, and those who survived less than 2 years as the STS. Surgery was defined as three styles, including primary surgery (primary sites surgery), curative surgery (primary sites and metastatic sites surgery) and metastatectomy (metastatic sites surgery).

\section{Statistical analysis}

The Chi-square test and the Fisher's exact probability test were used to compare demographic and clinicopathological variables between the LTS group and the STS group. Cox regression analysis was performed to evaluate prognostic factors. $\mathrm{p}<0.05$ was considered statistically significant, and the statistical tests were based on a two-sided significance level. A nomogram based on possible prognostic factors associated with OS was established using R software, on the basis of the Cox regression model. The performance of the nomogram was assessed with respect to discrimination and calibration. The discriminative abilities of prognostic models were evaluated with Harrell's concordance index (C-index). The C-index estimates the probability of concordance between the observed OS and OS that are predicted from the model. The value of the C-index statistic ranged from 0.5 to 1 , with 1 indicating perfect concordance, 0.5 indicating no better concordance than chance, and higher C-index values indicated a better prognostic model. Calibration was quantified by comparing the predicted OS with that of the observed survival against the nomogram's 1- and 2year predicted OS.

\section{Results}

A total of 2981 patients from the SEER database were included for analysis, of which 180 patients in the LTS group and 2801 in the STS group. The demographic and clinicopathologic variables were summarized in Table 1.

No significant difference in gender, age, race, $\mathrm{N}$ stage, location, histology, lung metastasis and brain metastasis was found between the two groups. The median age at diagnosis was 63 years and 65 years in the LTS group and the STS group, respectively. There was a significantly higher proportion of married patients in the LTS group compared to the STS group $(p=0.001)$. The LTS group had a higher proportion of well differentiated tumors $(41.1 \%$ vs $29.7 \%)$, whereas the STS group had a higher proportion of poorly differentiated tumors ( $58.9 \%$ vs $70.3 \%, p=0.001)$. SEER database provided information of surgery. A novel higher proportion of patients in the LTS group received surgery $(22.2 \%$ vs $7.0 \%, p<0.001)$. In the LTS group, 27 patients underwent primary surgery, 6 patients underwent curative setting surgery (both primary and metastatic sites surgery), and 7 patients underwent only metastectomy. In the STS group, 71 patients underwent primary surgery, 14 patients underwent curative setting surgery and 110 patients underwent only metastectomy.

The distribution of site-specific metastasis was different in the two groups. In the LTS group, 17 $(9.4 \%)$ patients had bone metastasis, 62 (34.4\%) patients had liver metastasis, $40(22.2 \%)$ patients had lung metastasis and $5(2.8 \%)$ patients had brain metastasis; while in the STS group, 659 (23.5\%) patients had bone metastasis, 1222 (43.6\%)patients had liver metastasis, $795(28.4 \%)$ patients had lung metastasis and $132(4.7 \%)$ patients had brain metastasis. The LTS group had significant lower proportions of bone metastasis $(p<0.001)$ and liver metastasis $(p=0.016)$, and a tendency of less lung metastasis $(p=0.077)$. However, the proportions of patients with brain metastasis $(p=0.229)$ were comparable between the two groups. Only $8.9 \%$ of the patients had multiple metastatic sites in the LTS group, while as high as $22.1 \%$ of the patients had multiple metastatic sites in the STS group $(p<0.001)$. 
Table 1. Demographic and clinicopathologic characteristics of the LTS group and the STS group

\begin{tabular}{|c|c|c|c|c|}
\hline & $\begin{array}{l}\text { Long time } \\
\text { survivals } \\
\text { (LTS) }\end{array}$ & $\begin{array}{l}\text { Shorter time } \\
\text { survivals (STS) }\end{array}$ & Total & P value \\
\hline Gender & & & & 0.470 \\
\hline Male & $147(81.7 \%)$ & $2342(83.6 \%)$ & 2489 & \\
\hline Female & $33(18.3 \%)$ & $459(16.4 \%)$ & 492 & \\
\hline Age & & & & 0.147 \\
\hline$<65$ & $98(54.4 \%)$ & $1369(48.9 \%)$ & 1467 & \\
\hline$\geq 65$ & $82(45.6 \%)$ & $1432(51.1 \%)$ & 1514 & \\
\hline Race & & & & 0.562 \\
\hline White & $157(87.2 \%)$ & $2361(84.3 \%)$ & 2518 & \\
\hline Black & $16(8.9 \%)$ & $296(10.6 \%)$ & 312 & \\
\hline Others & $7(3.9 \%)$ & $144(5.1 \%)$ & 151 & \\
\hline Marital status & & & & 0.001 \\
\hline Married & $124(68.9 \%)$ & $1584(56.6 \%)$ & 1708 & \\
\hline Unmarried & $56(31.1 \%)$ & $1217(43.4 \%)$ & 1273 & \\
\hline T stage & & & & 0.000 \\
\hline $\mathrm{T} 1$ & $51(28.3 \%)$ & $1008(36.0 \%)$ & 1059 & \\
\hline $\mathrm{T} 2$ & $18(10.0 \%)$ & $179(6.4 \%)$ & 197 & \\
\hline T3 & 79 (43.9\%) & $837(29.9 \%)$ & 916 & \\
\hline $\mathrm{T} 4$ & $32(17.8 \%)$ & $777(27.7 \%)$ & 809 & \\
\hline N stage & & & & 0.894 \\
\hline N0 & $43(23.9 \%)$ & $703(25.1 \%)$ & 746 & \\
\hline N1 & $107(59.4 \%)$ & $1595(56.9 \%)$ & 1702 & \\
\hline N2 & $20(11.1 \%)$ & $316(11.3 \%)$ & 336 & \\
\hline N3 & $10(5.6 \%)$ & $187(6.7 \%)$ & 197 & \\
\hline Location & & & & 0.683 \\
\hline Cervical & $2(1.1 \%)$ & $35(1.2 \%)$ & 37 & \\
\hline Thoracic & $159(88.3 \%)$ & $2369(84.6 \%)$ & 2528 & \\
\hline Upper & 8 & 95 & 103 & \\
\hline Middle & 17 & 325 & 342 & \\
\hline Lower & 129 & 1833 & 1962 & \\
\hline NOS & 5 & 116 & 121 & \\
\hline Abdominal & $1(0.6 \%)$ & $13(0.5 \%)$ & 14 & \\
\hline Overlapping & $8(4.4 \%)$ & $162(5.8 \%)$ & 170 & \\
\hline NOS & $10(5.6 \%)$ & $222(7.9 \%)$ & 232 & \\
\hline Histology & & & & 0.270 \\
\hline SCC & $39(21.7 \%)$ & $716(25.6 \%)$ & 755 & \\
\hline AC & $132(73.3 \%)$ & $1895(67.7 \%)$ & 2027 & \\
\hline Others & $9(5 \%)$ & $190(6.7 \%)$ & 199 & \\
\hline Grade & & & & 0.000 \\
\hline Grade I-II & $74(41.1 \%)$ & $833(29.7 \%)$ & 907 & \\
\hline Grade III-IV & $68(37.8 \%)$ & $1473(52.6 \%)$ & 1541 & \\
\hline Unknown & $38(21.1 \%)$ & $495(17.7 \%)$ & 533 & \\
\hline Bone metastasis & & & & 0.000 \\
\hline No & $163(90.6 \%)$ & $2142(76.5 \%)$ & 2305 & \\
\hline Yes & $17(9.4 \%)$ & $659(23.5 \%)$ & 676 & \\
\hline Brain metastasis & & & & 0.229 \\
\hline No & $175(97.2 \%)$ & $2669(95.3 \%)$ & 2844 & \\
\hline Yes & $5(2.8 \%)$ & $132(4.7 \%)$ & 137 & \\
\hline Liver metastasis & & & & 0.016 \\
\hline No & $118(65.6 \%)$ & $1579(56.4 \%)$ & 1697 & \\
\hline Yes & $62(34.4 \%)$ & $1222(43.6 \%)$ & 1284 & \\
\hline Lung metastasis & & & & 0.077 \\
\hline No & $140(77.8 \%)$ & $2006(71.6 \%)$ & 2146 & \\
\hline Yes & $40(22.2 \%)$ & $795(28.4 \%)$ & 835 & \\
\hline $\begin{array}{l}\text { Metastasis } \\
\text { numbers }\end{array}$ & & & & 0.000 \\
\hline $\begin{array}{l}\text { Oligometastatic } \\
\text { site }^{*}\end{array}$ & $164(91.1 \%)$ & $2182(77.9 \%)$ & 2346 & \\
\hline $\begin{array}{l}\text { Multiple } \\
\text { metastatic sites }\end{array}$ & $16(8.9 \%)$ & $619(22.1 \%)$ & 635 & \\
\hline Surgery & & & & 0.000 \\
\hline Yes & $40(22.2 \%)$ & $195(7.0 \%)$ & 235 & \\
\hline Primary surgery & 27 & 71 & 98 & \\
\hline Curative surgery & 6 & 14 & 20 & \\
\hline Metastatectomy & 7 & 110 & 117 & \\
\hline No & $140(77.8 \%)$ & $2606(93.0 \%)$ & 2746 & \\
\hline Total & 180 & 2801 & 2981 & \\
\hline
\end{tabular}

Multivariate analysis demonstrated that gender, race, histology, marital status, $\mathrm{T}$ stage, grade, bone metastasis, brain metastasis, lung metastasis, liver metastasis, and primary surgery were independent prognostic factors for 1-year OS and 2-year OS. However, surgery (including primary surgery, curative setting surgery and metastectomy) was not one of the independent prognostic factors.

A nomogram incorporating all significant independent factors for predicting 1-year OS and 2-year OS was established based on selected variables with hazard ratios (Figure 1). Each variable was given a score on a points scale. By adding up the total scores projected in the bottom scale, we could estimate the probability of 1- and 2-year OS. Based on intern validation, the Harrell's C-index for 1-year and 2-year OS prediction was 0.633 (95\% CI: 0.608-0.658). The calibration plot for the probability of OS at 1 or 2 years showed a good correlation between the nomogram prediction and actual observation (Figure 2).

\section{Discussion}

In our study, we compared the clinicopathologic characteristics between the long-time survivals and the short time survivals, and found that married patients, patients with well differentiated tumors, patients without bone metastasis or liver metastasis and those who underwent primary surgery are associated with long time survivals.

Marital status plays an important role in patients' survival of various cancers [4-5]. A Swedish study demonstrated that unmarried patients diagnosed of gastric cancer or esophageal cancer had poorer survival compared with the married patients [6]. Two recent population-based studies also found that marital status was associated with superior survival in patients with esophageal cancer (EC) [7-8]. On the contrary, Brusselaers $\mathrm{N}$ et al showed no evidence of a better 5-year survival in married patients with EC who received surgery [9]. However, in the study, only $11.4 \%$ of the patients had M1 diseases. It is reported that sicker patients including those with advanced cancer were less likely to marry and have a higher risk of dissolution of marriage [10]. To date, few studies focusing on marital status of advanced EC patients has been published. In our study, we found that there was a significantly higher proportion of married patients in the LTS group compared to the STS group. Also, multivariate analysis showed that marital status was one of the prognostic factors for metastatic EC patients. Better social and spiritual support, improved nutrition condition bought by good marriage may contribute to better survival. 


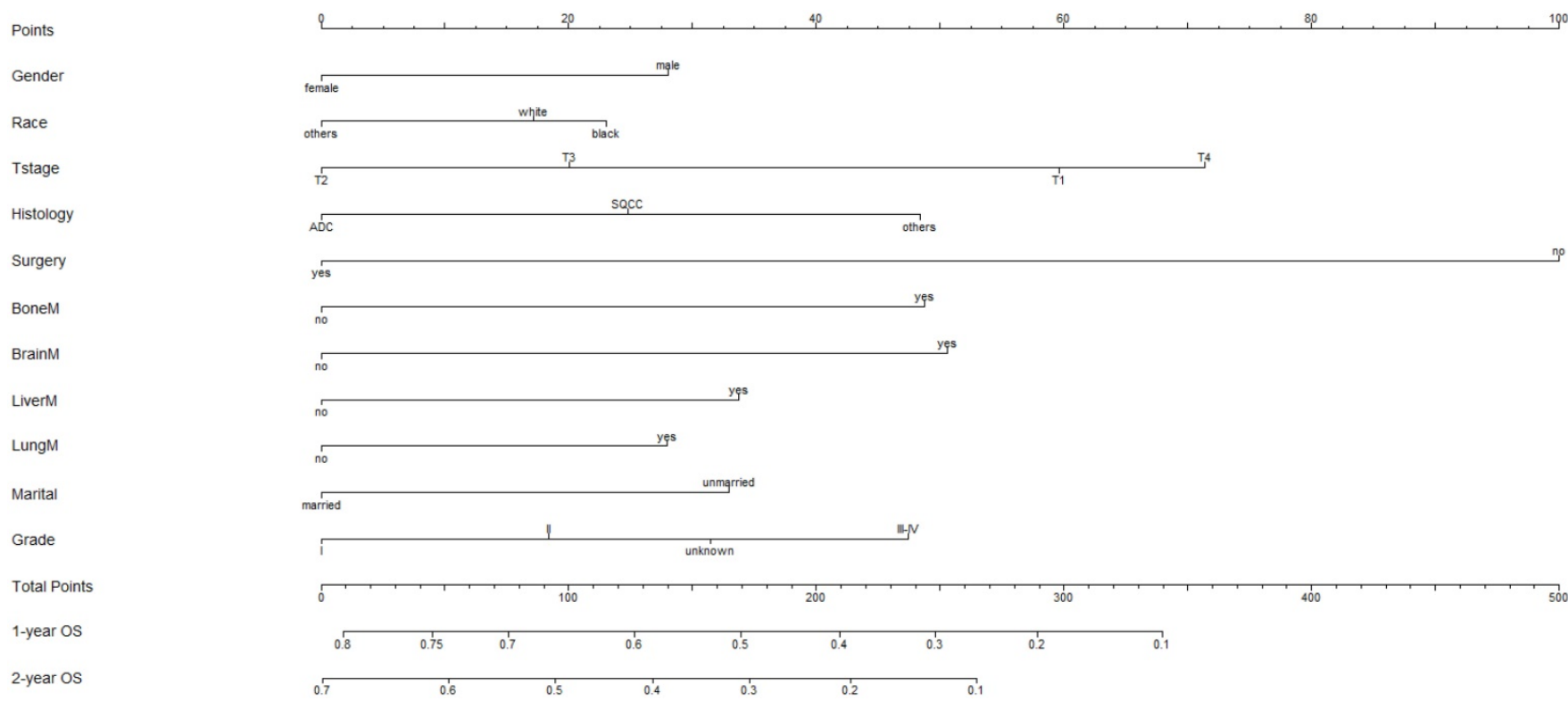

Figure 1. Nomogram to predict the probability of 1-year OS and 2-year OS

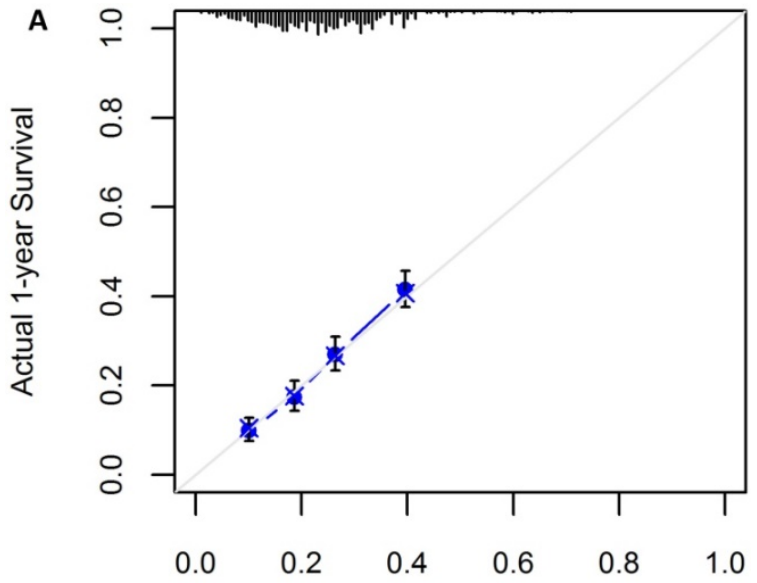

Nomogram Predicted 1-year Survival

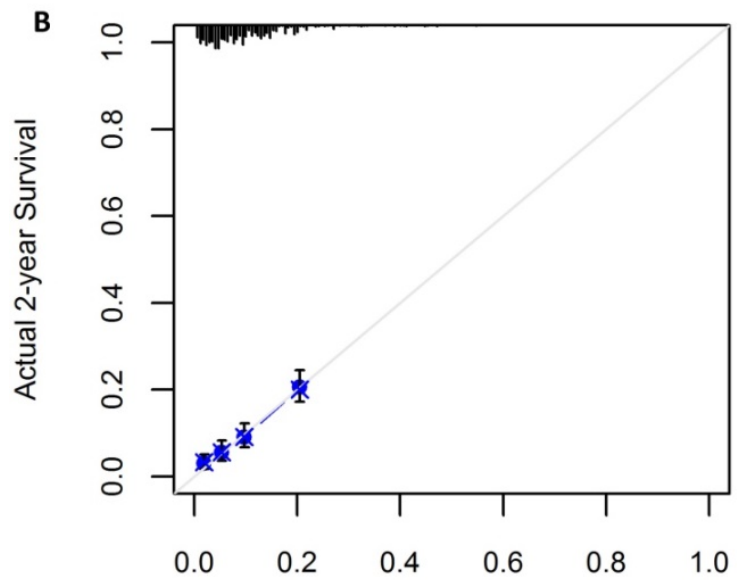

Nomogram Predicted 2-year Survival

Figure 2. Calibration plot showing nomogram-predicted 1-year OS probabilities with the actual 1-year OS (A) and the nomogram-predicted 2-year OS with the actual 2-year OS (B)
Brain metastasis is rare in esophageal cancer, and the survival is poor. The median survival was 4 months and the 1-year OS was $18 \%$ as published [11]. However, in our study, no significant difference in brain metastasis was found between the LTS group and the STS group $(p=0.164)$. Some of the patients can achieve long-time survival even if they had brain metastasis. Onal $\mathrm{C}$ et al reported the survival condition of seven patients with solitary brain metastasis. All but one patient received definitive chemoradiothearapy and whole-brain radiotherapy. The median survival time was 18.9 months (range: 10.0-27.2 months) [12]. Welch $G$ et al found that for esophageal cancer patients with brain metastasis, OS was superior for those initially had surgical resection with the 1-year OS as high as $67 \%$ [11]. It is indicated that although brain metastasis is one of the prognostic factors for poor survival, those who have solitary metastasis or who can tolerate aggressive treatments may have long-time survivals.

In our study, we found that primary surgery was an independent prognostic factor for stage IV EC. Compared to patients with primary surgery, the hazard ratios for patients who didn't receive surgery was 1.922 (95\% CI: 1.524-2.425). Also, a novel higher proportion of patients in the LTS group received primary surgery $(18.3 \%$ vs $3 \%, p<0.001)$. Currently, National Comprehensive Cancer Network (NCCN) guidelines recommend chemotherapy as the standard treatment for stage IV disease of EC. Surgery is considered as a palliative treatment and the role of surgery in stage IV diseases is controversial. Blank $\mathrm{S}$ et al observed long-term survival when they studied 160 patients with metastatic esophagogastric adenocarci- 
noma who were treated with chemotherapy followed by resection of the primary tumor and metastasis [13]. Wang J et al reported 96 patients with stage IVB EC treated with chemotherapy followed by concurrent chemoradiation \pm surgery, the median OS time was 21 months, and 2-year OS rate was $46.8 \%$. In the study, 14 patients received surgery, and the median OS was significantly superior compared to patients without surgery $(p=0.001)[14]$. However, Saddoughi SA et al recommended esophagectomy not performed for stage IV EC patients in another retrospective study for 52 stage IV EC patients who underwent radical surgical resection [15]. In our study, primary surgery was one of the independent prognostic factors, however, surgery including all the three styles (primary surgery, curative surgery and metastectomy) was not. It indicated that primary surgery may be associated with more benefits. Still, further work, especially prospective study with a large population, is warranted to evaluate the value of surgery in stage IV EC.

This study has several limitations. First, it is a retrospective study which had unavoidable selection bias. Second, other metastatic sites apart from brain, bone, liver and lung, the use of chemotherapy or radiotherapy and surgery details are not reported in the SEER database. Furthermore, we did not perform an external validation, so concerns about generalizability are warranted. Therefore, the presented nomogram needs to be replicated and then prospectively validated before it can be implemented in clinical practice.

\section{Conclusions}

In conclusion, married patients, patients with well differentiated tumors, patients with oligometastatic site, patients without bone metastasis or liver metastasis and those who underwent surgery are associated with long time survivals. We developed a nomogram predicting 1- and 2-year OS and CSS for M1 stage esophageal cancer. The prognostic model may improve clinicians' abilities to predict individualized survival and to make treatment recommendations.

\section{Acknowledgments}

We would like to thank all the physicians and physicists in Department of Radiation Oncology, Shanghai Chest Hospital, Shanghai Jiao Tong University for their assistance in the research.

This work was supported by the National Natural Science Foundation of China (grant 81502645).

\section{Authors' contribution}

Xuwei Cai designed the study and supervised the research work. Mina Liu and Lanting Gao collected data. Changlu Wang did the statistical analysis. Mina Liu wrote the article and Changxing Lv revised it.

\section{Competing Interests}

The authors have declared that no competing interest exists.

\section{References}

1. Torre LA, Bray F, Siegel RL et al. Global cancer statistics. 2012. CA Cancer J Clin. 2015; 65: 87-108.

2. Arnold M, Soerjomataram I, Ferlay J et al. Global incidence of oesophageal cancer by histological subtype in 2012. Gut. 2015; 64 (3): 381-7.

3. Tanaka T, Fujita H, Matono $\mathrm{S}$ et al. Outcomes of multimodality therapy for stage IVB esopahageal cancer with distant organ metastasis. Dis Esophagus. 2010; 23 (8): 646-51.

4. Aizer AA, Chen $\mathrm{MH}$, McCarthy EP et al. Marital status and survival in patients with cancer. J Clin Oncol. 2013; 31:3869-76.

5. Li Q, Gan L, Liang L et al. The influence of marital status on stage at diagnosis and survival of patients with colorectal cancer. Oncotarget. 2015; 6: 7339-47.

6. Ljung R, Drefahl S, Andersson G et al. Socio-demographic and geographical factors in esophageal and gastric cancer mortality in Sweden. PLoS One. 2013; 8: e62067.

7. Du L, Kim JJ, Chen B et al. Marital status is associated with superior survival in patients with esophageal cancer: a Surveillance, Epidemiology, and End Results study. Oncotarget. 2017; 8 (56): 95965-72.

8. Zhang $\mathrm{Q}$, Lin $\mathrm{X}$, Zhang $\mathrm{C}$ et al. The influence of marital status on the survival of patients with esophageal cancer: a population-based, propensity-matched study. Oncotarget. 2017; 8 (37): 62261-73.

9. Brusselaers N, MKattsson F, Johar A et al. Marital status and survival after oesophageal cancer surgery: a population based nationwide cohort study in Sweden. BMJ Open. 2014; 4 (6): e005418.

10. Wiiliams K, Umberson D. Marital status, marital transitions, and health: a gendered life course perspective. J Health Soc Behav. 2004; 45:81-98.

11. Welch G, Ross HJ, Patel NP et al. Incidence of brain metastasis from esophageal cancer. Dis Esophagus. 2017; 30(9):1-6.

12. Onal C, Akkus Yildrim B, Guler OC. Outcomes of aggressive treatment in esophageal cancer patients with synchronous solitary brain metastasis. Mol Clin Oncol. 2017; 7(1): 107-12.

13. Blank S, Lordick F, Dobritz $\mathrm{M}$ et al. A reliable risk score for stage IV esophagogastric cancer. Eur J Surg Oncology. 2013; 39(8): 823-30.

14. Wang J, Suri JS, Allen PK et al. Factors predictive of improved outcomes with multimodality local therapy after palliative chemotherapy for stage IV esophageal cancer. Am J Clin Oncol. 2016: 39 (3): 228-35.

15. Saddoughi SA, Reinersman JM, Zhukov YO et al. Survival after surgical resection of stage IV esophageal cancer. Ann Thorac Surg. 2017; 103 (1): 261-66. 